OPEN ACCESS

Edited by:

Tao Jiang,

Capital Medical University, China

Reviewed by:

Kristin Huntoon,

University of Texas MD Anderson

Cancer Center, United States

Jeremie Vitte,

University of California, Los Angeles,

United States

*Correspondence:

Marco Crocco

marcocrocco@gaslini.org

tThese authors have contributed equally to this work and share first

authorship

Specialty section:

This article was submitted to Neuro-Oncology and Neurosurgical

Oncology,

a section of the journal

Frontiers in Neurology

Received: 14 July 2021 Accepted: 04 October 2021 Published: 29 October 2021

Citation:

Crocco M, Panciroli M, Milanaccio C,

Morerio C, Verrico A, Garrè ML, Di

lorgi N and Capra V (2021) Case

Report: The Emerging Role of Ring

Chromosome 22 in Phelan-McDermid

Syndrome With Atypical

Teratoid/Rhabdoid Tumor: The First

Child Treated With Growth Hormone.

Front. Neurol. 12:741062

doi: 10.3389/fneur.2021.741062

\section{Case Report: The Emerging Role of Ring Chromosome 22 in Phelan-McDermid Syndrome With Atypical Teratoid/Rhabdoid Tumor: The First Child Treated With Growth Hormone}

\author{
Marco Crocco ${ }^{1,2 * t}$, Marta Panciroli ${ }^{2 \dagger}$, Claudia Milanaccio ${ }^{1}$, Cristina Morerio ${ }^{3}$, \\ Antonio Verrico ${ }^{1}$, Maria Luisa Garrè ${ }^{1}$, Natascia Di lorgi ${ }^{2,4}$ and Valeria Capra ${ }^{5}$ \\ ${ }^{1}$ Neuro-Oncology Unit, Istituto di Ricovero e Cura a Carattere Scientifico Giannina Gaslini Institute, Genoa, Italy, \\ ${ }^{2}$ Department of Neuroscience, Rehabilitation, Ophthalmology, Genetics, Child and Maternal Health, University of Genova, \\ Genoa, Italy, ${ }^{3}$ Laboratory of Human Genetics, Istituto di Ricovero e Cura a Carattere Scientifico Giannina Gaslini Institute, \\ Genoa, Italy, ${ }^{4}$ Department of Pediatrics, Istituto di Ricovero e Cura a Carattere Scientifico Giannina Gaslini Institute, Genoa, \\ Italy, ${ }^{5}$ Medical Genetics Unit, Istituto di Ricovero e Cura a Carattere Scientifico Giannina Gaslini Institute, Genoa, Italy
}

Atypical teratoid/rhabdoid tumors (AT/RTs) in the rhabdoid tumor predisposition syndromes are most often caused by germline mutations of the SMARCB1 gene located in chromosome 22q11.2. Although rarely, it can also result from the constitutional ring chromosome 22 (r22): during mitosis the ring chromosome may lead to an increased rate of somatic mutations, resulting in rhabdoid tumor predispositions when the tumor-suppressor gene SMARCB1 is involved. Individuals with r22 may present similar features as those with Phelan-McDermid syndrome (PMDS) due to 22q13.3 deletion, including the SHANK3 gene. Despite several reports on AT/RT in children with r22 and/or PMDS have been published, the role of constitutional r22 as new oncogenic mechanism for AT/RT is still under investigation. There is not a lot of data available on therapeutic and prognostic implications of r22 in AT/RT and PMDS. Herein, we present the first case of a child with constitutional r22, PMDS and AT/RT of the brain, who is a long term survivor and is been treated with growth hormone. We also describe an unexpected adverse reaction to midazolam.

Keywords: atypical teratoid/rhabdoid tumor, growth hormone, SMARCB1, INI1, ring chromosome, midazolam, case report, Phelan McDermid Syndrome (PHMDS)

\section{INTRODUCTION}

Atypical teratoid/rhabdoid tumors (AT/RTs) represent $20 \%$ of highly malignant tumors of the central nervous system (CNS), in children $<3$ years old (1).

The SMARCB1/INI1 gene codes a subunit of the switch/sucrose non-fermentable (SWI/SNF) complex, actin-dependent chromatin remodeling complex and is known to act as a tumor suppressor. This gene is frequently inactivated through somatic point mutation or deletion, followed by a second hit. The constitutional pathogenic variant in SMARCB1 causes rhabdoid tumor predisposition syndrome. About $25 \%-35 \%$ of newly diagnosed children with rhabdoid 
tumors have a germline pathogenic variant in SMARCB1 (2). The rhabdoid tumor predisposition syndrome is rarely as a consequence of the loss of the expression of the ATPase subunit SMARCA4, also known as BRG1, another SWI/SNF chromatin-remodeling complex member (3). Therefore, the presence of the expression of SWI/SNF-related matrix-associated actin-dependent regulator of chromatin subfamily B member 1 (SMARCB1) protein does not rule out a diagnosis of AT/RT (4).

AT/RT has been previously reported in patients with r22 (58) and recently also in PMDS (OMIM\# 606232) (9-11). Ring chromosome 22 syndrome is an autosomal anomaly with most features common to $22 \mathrm{q} 13$ deletion syndrome. Byers et al. (11) hypothesized that mitotic instability of $\mathrm{r} 22$ could lead to somatic mosaic aneuploidy, resulting in a heterozygous loss of SMARCB1. A complete loss of $S M A R C B 1$ expression in tumor cells can be the result of a second-hit or an alternative inactivating mechanism, such as methylation pattern, variants in non-coding regions, promoters or enhancers.

PMDS is a contiguous gene disorder caused by a heterozygous contiguous gene deletion at chromosome $22 \mathrm{q} 13$ or by mutation in the SHANK3 gene, which is located within the minimum critical region. The loss of 22q13.3 can result from a simple deletion, translocation, ring chromosome formation or, less commonly, from structural changes affecting the long arm of chromosome 22, specifically the region containing the SHANK3 gene. It is underdiagnosed and till now the prevalence is unknown (12). More than $50 \%$ of patients show autism or autisticlike behavior due to haploinsufficiency of the synaptic scaffolding protein SHANK3. Other major features of PMDS include: global developmental delay, neurologic and motor regression with moderate to severe intellectual impairment, absent or severely delayed speech, hypotonia, dysmorphic features, normal to accelerated growth and increased pain tolerance (13).

In cellular models of PMDS, neurons with reduced SHANK3 expression show defects in excitatory, but not inhibitory, synaptic transmission. Excitatory synaptic transmission can be corrected by restoring SHANK3 expression or by treating neurons with insulin-like growth factor 1 (IGF-1) leading to the formation of mature excitatory synapses that lack SHANK3 but contain the post synaptic density protein-95 (PSD-95) and N-methyl-Daspartate (NMDA) receptors.

In the literature, patients with autism spectrum disorders or PMDS do not show an increased incidence of adverse events post-procedural sedation with midazolam $(14,15)$. Li et al. have examined whether sensitivity to isoflurane anesthesia is altered in genetic mouse model of PMDS (Shank3 deleted), they showed that Shank3 deletion confers enhanced sensitivity to isoflurane (16).

Herein, we present from a multidisciplinary perspective a complex case of r22 in a child with PMDS due to a partial deletion of chromosome 22 not involving the SMARCB1 gene, who survived a metastatic AT/RT of the CNS. We also describe, for the first time in such a patient, the benefits of hrGH therapy and a serious adverse event after procedural sedation with midazolam.

\section{CASE PRESENTATION}

The child was born after a 38-week uncomplicated pregnancy from non-consanguineous parents. The family history was uneventful. APGAR (Appearance, Pulse, Grimace, Activity, Respiration) scores were 9 at the 1 st $\min$ and 10 at 5 th min. After delivery, the neonate did not present major dysmorphisms or hypotonia; normal growth and development were reported (Figures 1, 2).

At 2 years and 6 months the child started having feeding problems and vomiting episodes and he underwent a brain Magnetic Resonance Imaging (MRI) that showed diffused pathological tissue of the 4 th ventricle area with metastatic

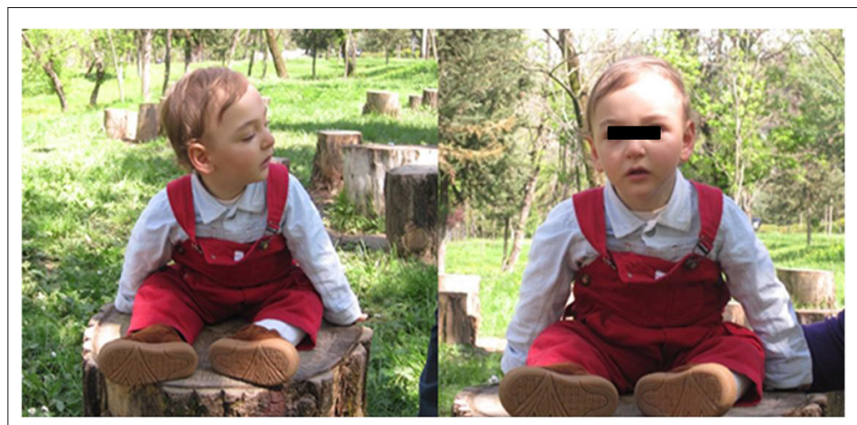

FIGURE 1 | One year old.

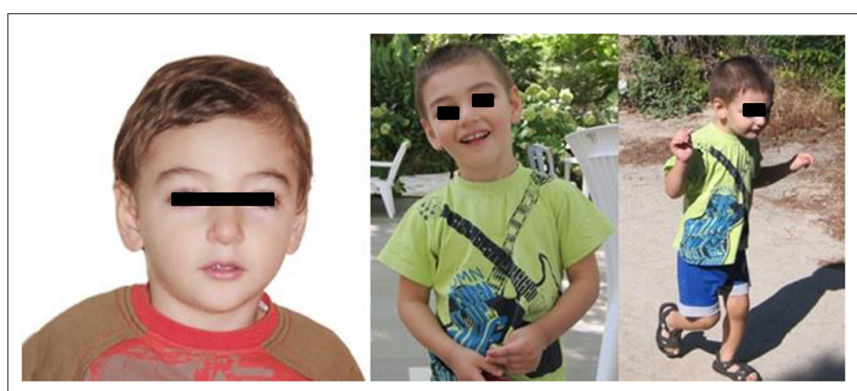

FIGURE 2 | Two years old.

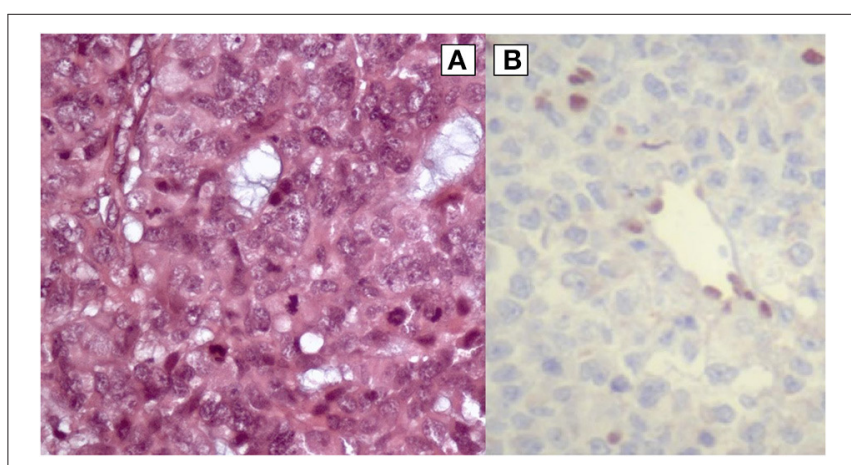

FIGURE 3 | (A) High-power view illustrates AT/RT cells of the patient (hematoxylin and eosin staining). (B) Rhabdoid cells negative for SMARCB1 protein (immunohistochemistry). 
diffusion at the left temporal brain lobe, spinal cord at D5-D6 level and cauda.

Partial resection of the mass occupying the 4 th ventricle allowed the diagnosis of AT/RT. Histological analysis of the tumor showed a population of cells with rhabdoid features (Figure 3A). Immunohistochemistry showed the absence of the expression of SMARCB1 protein (Figure 3B), at the same time the expression of EMA and focal of AML and SYN was observed (data not shown). The histological diagnosis was confirmed by a national centralized reviewer. An abdominal ultrasound ruled out the presence of any renal mass.

After neurosurgery, the patient's clinical conditions were very severe due to the progression of the spinal metastasis, with pain difficult to control, progressive paraparesis, sphincter impairment and posterior fossa syndrome. Chemotherapy (CT) was started according to European protocol EU RHAB 2010 and adapted for the critical clinical conditions. Three courses of induction CT (Doxorubicin $1.4 \mathrm{mg} / \mathrm{kg} / 24 \mathrm{~h} \times 2$ days; Vinorelbine $0.7 \mathrm{mg} / \mathrm{kg}$ for 3 days; 2 ICE cycles: Ifosfamide $66.6 \mathrm{mg} / \mathrm{kg}$ and Etoposide $3.3 \mathrm{mg} / \mathrm{kg}$ x 3 days, Carboplatinum $16.7 \mathrm{mg} / \mathrm{kg}$ for 1 day) and 2 courses of myeloablative high dose CT (1 course of Thiotepa $300 \mathrm{mg} / \mathrm{m}^{2}$ x 3 days + Carbo $500 \mathrm{mg} / \mathrm{m}^{2}$ x 3 days and 1 course of Thiotepa $300 \mathrm{mg} / \mathrm{m}^{2} \times 3$ days) were delivered. The MRI showed that all the sites exhibited a good response after the induction-and myeloablative-CT. Treatment was completed at the age of 3 years and 2 months when the child had been irradiated by Tomotherapy technique: $3520 \mathrm{cGy}$ on the cranio-spinal axis, followed by boosts on sites of residual disease: posterior fossa and temporo-polar lesions up to $5800 \mathrm{cGy}$; cauda equina up to 4960 cGy; D5-D6 4960 up to cGy.

After irradiation, the child suffered prolonged leukopenia and thrombocytopenia with one episode of general infection treated with multiple transfusions and broad-spectrum antibiotics. Valproic Acid was chosen as a prophylactic anti-seizure drug for its anti-tumor properties in high-grade brain tumors $(17,18)$.

The MRI at 1 and 3 months after irradiation showed a complete remission at the level of the primary site, while millimetric cystic lesions improved slightly (with signal like cerebrospinal fluid) at the temporo-polar site and at the residual spinal lesions. The MRI, 6 months after the therapies, showed a definite improvement of the metastatic spinal lesions, which were no longer detectable.

Despite starting the intensive multidisciplinary rehabilitation program early, the patient exhibited the following signs: growth and weight impairment; severe psychomotor regression; global hypotonia; cognitive impairment with only partial recovery after rehabilitation; oropharyngeal dysphagia for liquid and solid food; osteoporosis and low trauma vertebral fractures; and alopecia.

Three years after the end of radiotherapy, the child had a complete neuroradiologic remission of the disease, and the patient met auxological criteria to initiate evaluation for growth hormone deficiency (GHD). Even though the height was appropriate for age, sex and target height, it dropped from +0.8 to-0.3 SDS (Standard Deviation Score) due to the pathological growth velocity ( $<2 \mathrm{~cm}$ in 1 year). Two provocative $\mathrm{GH}$ tests confirmed the diagnosis of GHD (the peak value of serum GH was $3,52 \mathrm{ng} / \mathrm{mL}$ after stimulation with intravenous infusion of
Arginine, and $6.63 \mathrm{ng} / \mathrm{mL}$ after stimulation with intramuscular injection of Glucagone; in both tests the normal values were $>8 \mathrm{ng} / \mathrm{ml}$ ). A full evaluation of the other pituitary hormones ruled out further damages. Recombinant human $\mathrm{GH}$ (hrGH) was started at a $0.028 \mathrm{mg} / \mathrm{kg} /$ day dose without adverse effects. After 6 months of hrGH, the 1 year growth velocity increased from $4 \mathrm{~cm} /$ year $(-2.2 \mathrm{SDS})$ to $5.5 \mathrm{~cm} /$ year $(-0.3 \mathrm{SDS})$ and the parents reported an improved quality of life, muscle strength and physical capability. At the last evaluation, after 18 months of treatment, the growth velocity remained consistently regular $(6.2$ $\mathrm{cm} /$ year, $-0.8 \mathrm{SDS}$ ); IGF-1 increased from $47.6 \mathrm{ng} / \mathrm{ml}$ (-3.8 SDS) to $78.1 \mathrm{ng} / \mathrm{ml}(-2.3 \mathrm{SDS}) 6$ months after hrGH initiation $(0.028$ $\mathrm{mg} / \mathrm{kg} /$ day), reaching normal values up to $146.2 \mathrm{ng} / \mathrm{ml}(-0.55$ SDS) after a further 18 months of treatment $(0.031 \mathrm{mg} / \mathrm{kg} /$ day $)$. The latter dose is currently ongoing without side effects.

As for institutional protocol, intranasal midazolam was implemented to increase the patient's compliance to procedural sedation for MRI; shortly after midazolam was administered for the first time to our patient (months before starting hrGH) he showed signs of shock (severe hypotension, prolonged capillary refill time, a decreased level of consciousness and urinary output, and bradycardia) and needed resuscitation. The hypovolemic shock treatment included three intravenous boluses of fluids 20 $\mathrm{ml} / \mathrm{kg}$ ( $5 \%$ dextrose in $\mathrm{NaCl} 0.9 \%$ physiological solution), and a hydrocortisone $10 \mathrm{mg} / \mathrm{kg}$ intravenous injection which resulted in a slow recovery of the state of consciousness and improvement of the vital signs. A subsequent ACTH (adrenocorticotropic hormone) low dose test excluded central adrenal insufficiency. An MRI was performed 5 months after starting hrGH using Propofol intravenously as procedural sedation without side effects. Complete remission of the disease was confirmed after 18 months of hrGH therapy ( 5 years after the end of oncological treatment).

Mild dysmorphic facial features were detectable by facial photos before chemo-radiotherapy (long eyelashes, bulbous nose, large and prominent ears) (Figure 2) and due to the severe late effects (Figure 4) the child underwent genetic analysis. ArrayCGH (Whole-genome $60 \mathrm{~K}$ Agilent array, Human Genome CGH Microarray, Agilent Technologies, Santa Clara, CA, USA) revealed a de-novo chromosomal rearrangement: $\operatorname{arr}$ [GRCh37] 22q13.32q13.33(48746241_tel)x1 dn involving a partial deletion of long arm of chromosome 22 that is extended for $2,4 \mathrm{Mb}$ from nucleotide 48.746.241 to the telomere (Figure 5). Data was analyzed using Agilent Cytogenomics Software. All genomic positions were reported according to the human genome assembly (GRCh37/hg19). This genomic region is known to include 36 genes (including SHANK3) and this chromosomic region is responsible for the deletion syndrome of $22 \mathrm{q} 13.3$. The presence of the SHANK3 deletion confirmed the diagnosis of PMDS. SMARCB1 was not mutated in the germline DNA as demonstrated by the Next-Generation Sequencing analysis (Ion Torrent NGS sequencing systems, Ion AmpliSeq kit, Waltham, MA, USA), the MLPA analysis was not possible due to the lack of biological material available. Cytogenetic analysis using Q-banding techniques at the 400 bands of resolution revealed a 46, XY, r22(p11.2;q13.32)dn karyotype in the 30 metaphase cells examined (Figure 6A). FISH studies were performed using 


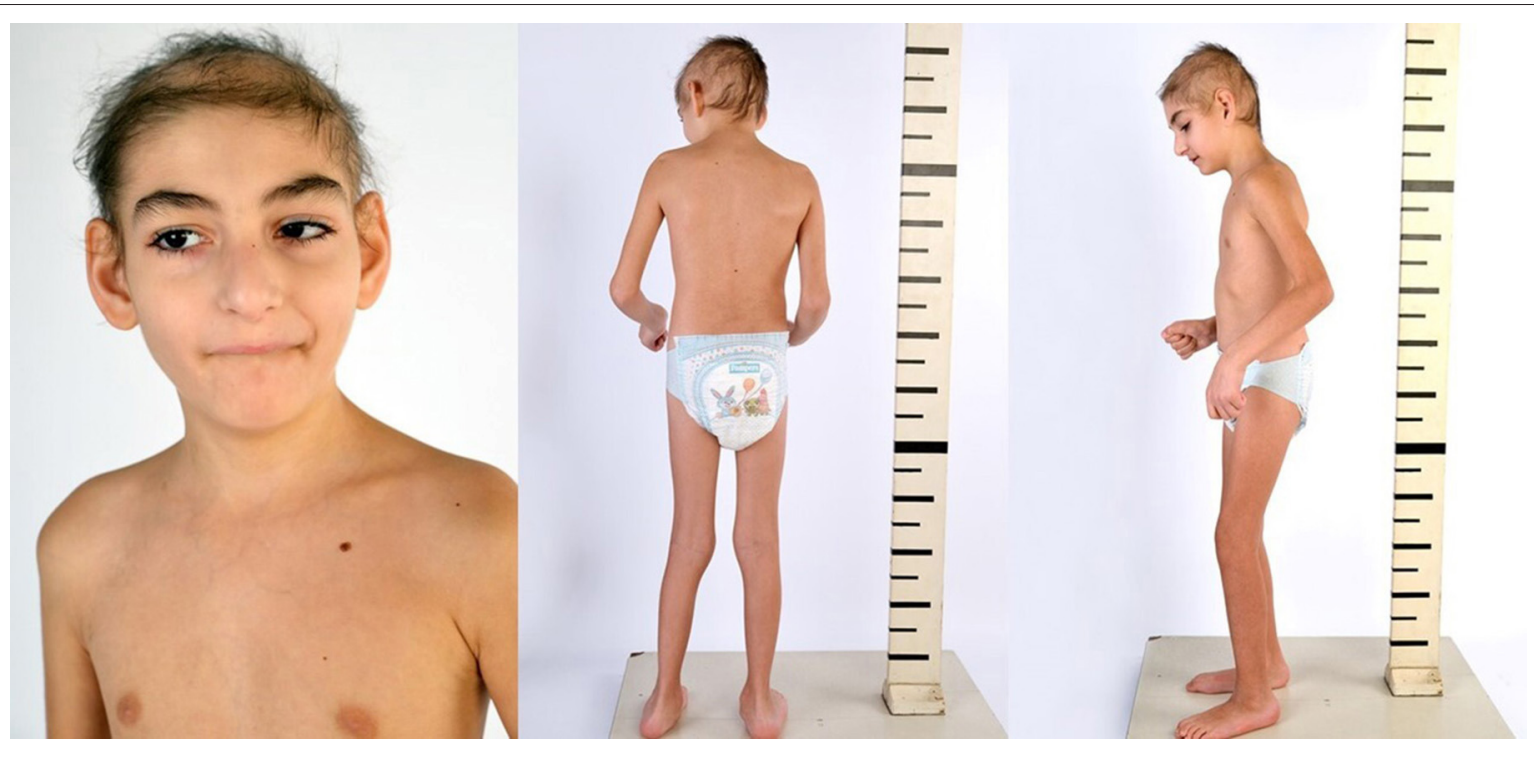

FIGURE 4 | Eight years old.

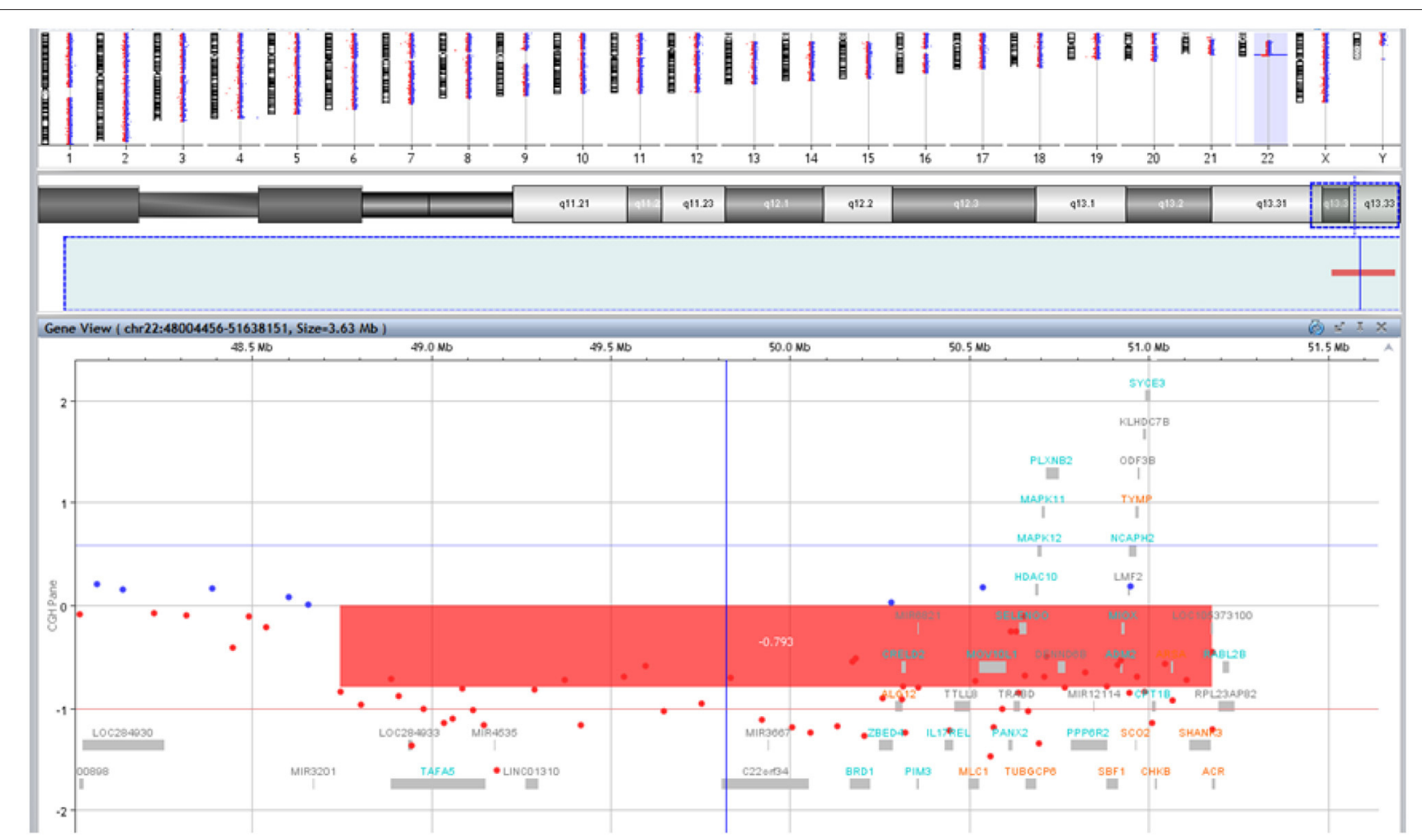

FIGURE 5 | Results of array-CGH analysis. Zoom view of long arm of chromosome 22 shows a 2,4 Mb deletion at 22q13.32q13.33 spanning from position 48746241 bp to the telomere.

SureFISH probe Agilent (Santa Clara, CA, USA) 22q13.33 SHANK3 and chromosome 14/22 alpha satellite probe Acquarius Cytocell (Cambridge, UK) (Figure 6B), the results confirmed the $\mathrm{CGH}$ Microarray and karyotype analysis. The genetic study of both parents was negative.

\section{DISCUSSION}

In our clinical case SMARCB1 was not mutated in the germline DNA and the partial deletion of chromosome 22 not involving the SMARCB1 gene, nevertheless the immunohistochemistry of 


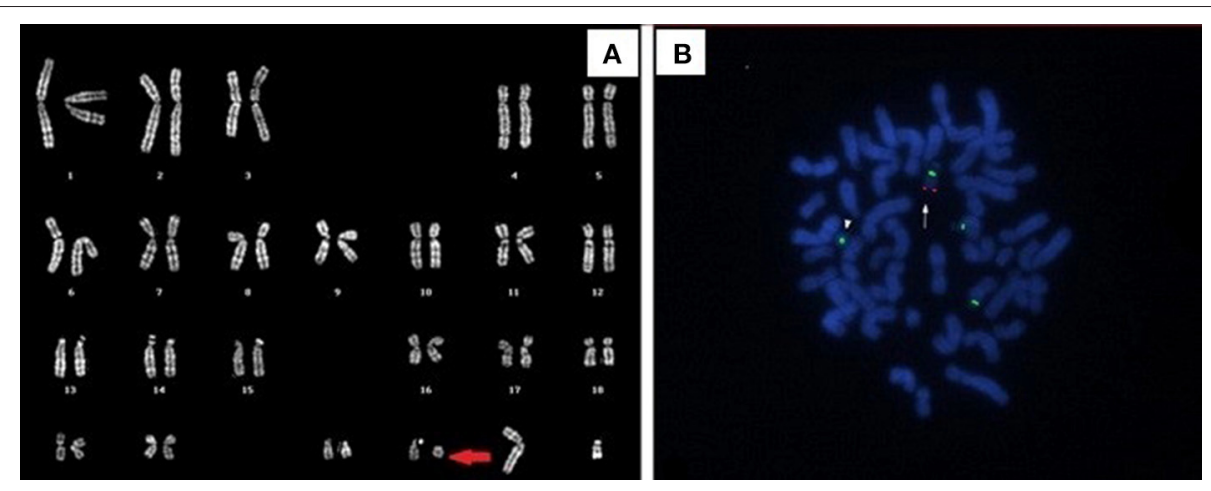

FIGURE 6 | (A) Q-banded karyotype showing ring chromosome 22. (B) FISH analysis with 14/22 centromeric probe and specific SHANK3 probe (green and red signals, respectively). The arrow indicates the normal chromosome 22, the arrowhead shows the absence of SHANK3 signal on the ring chromosome 22.

the AT/RT tumor of our patient demonstrated the absence of the SMARCB1 protein (Figure 3B), suggesting the loss of both alleles. We hypothesized that in cancer cells the loss of expression of the SMARCB1 protein was due to a somatic mosaic aneuploidy involving $\mathrm{r} 22$ and including $S M A R C B 1$, as described by Byers et al. (11), followed by a second-hit.

Familial cases described as the rhabdoid tumor predisposition syndrome, have been linked to heterozygous SMARCB1 germline mutations. The loss of expression of SMARCB1 is the defining molecular genetic feature of $\operatorname{AT} / \mathrm{RT}(19,20)$. Immunohistochemical staining of SMARCB1 is currently considered very sensitive and highly specific for the detection of SMARCB1 genetic defects (21). Tsai et al. (22), by using gene expression microarray analysis on Taiwanese AT/RT, found that four of the five AT/RT cases examined still showed positive SMARCB1 mRNA signal even though SMARCB1 proteins were stained negative by immunohistochemistry. They sequenced the whole SMARCB1 gene in 4 AT/RT cases (no DNA available for the fifth case) to check DNA mutations in the SMARCB1 gene genomic DNA region. Three of the four AT/RTs did not show sequence alternation in the SMARCB1 gene after the PCR-amplified genomic DNAs were isolated from fresh frozen tumor tissue. Similarly, in another independent cohort containing 20 AT/RTs, about $10 \%$ of cases did not have coding sequence mutations in any of the 9 exons of SMARCB1 and yet had decreased expression levels of SMARCB1 by RT-PCR analysis and undetectable levels of the protein by Western blot analysis (23).

The monosomy of $22 \mathrm{q} 13.3$ has findings typical of such deletion syndrome characterized by global developmental delay, generalized hypotonia, and absent or severely delayed speech (24), but the true prevalence of $22 \mathrm{q} 13.3$ deletion may be underestimated (12). The deleted region contains numerous genes that could have an effect on the presence of AT/RT and the developmental delay of the child.

Among the genes, the bromodomain containing 1 (BRD1) gene has been shown to be associated with schizophrenia and bipolar disorder in genetic studies, including genewise significant association in a large schizophrenia genomewide association study meta-analysis $(25,26)$. Furthermore,
Cai QQ et al. demonstrated that sulfatide interaction with the BRD1 mediates acetylation and is important for the regulation of integrin $\alpha \mathrm{V}$ gene expression in hepatocarcinoma (27) and similarly could have the same effect in AT/RT. While proviral integration of Moloney murine 3 (PIM3) is associated with Distal Muscular Dystrophy, it has also been demonstrated to function as an oncogenic factor promoting tumor growth in colorectal cancer (28) and hepatoblastoma (29). The modulator of VRAC current 1 (MLC1) is associated with Megalencephalic Leukoencephalopathy with subcortical cysts and it could be related to melanoma survival and chemoresistance through mitogen-activated protein kinases $(M A P K)$ - extracellular signal-regulated kinases (ERK) pathway $(30,31)$. The histone deacetylase 10 (HDAC10) is involved in transcriptional repression of the histone acetyl transferase $(H A T)$. In many cancers, the balance between HAT and HDAC is altered. Diseases associated with HDAC10 include Neuroblastoma (32), renal cell carcinoma (33) and lung adenocarcinoma (34). HDAC inhibitors (HDACis) were shown to exert antitumor effects in several cancer cell lines, confirming the role of HDACs in oncogenesis (35). MAPK8IP2 gene encodes a scaffold protein: The JNK-interacting protein (JIP), that is more abundantly expressed in the cerebellum, pituitary gland, occipital lobes and the amygdala, and it could be associated to Ameloblastic Carcinoma and Spinocerebellar Ataxia X-Linked 5. The p38-MAPK signaling, including p38 $\beta$ (MAPK11) and p38 $\gamma$ (MAPK12), is associated with the development and progression of several types of cancer (36-38). On the other hand, the MAPK pathway could be involved in the occurrence of AT/RT $(39,40)$. PLXNB2 is required for normal differentiation and migration of neuronal cells during brain corticogenesis and for normal embryonic brain development (41-43). Therefore, also PLXNB2 could contribute to the delay in developmental of the child.

SHANK3 is a protein coding gene and a member of the SHANK gene family. The SHANK3 protein is particularly involved in the postsynaptic density that connects neurotransmitter receptors, ion channels, and other membrane proteins to the actin cytoskeleton and G-protein-coupled signaling pathways. Mutations in the SHANK3 gene are a cause of autism spectrum disorder, which is characterized by 
impairments in social interaction and communication, and restricted behavioral patterns and interests. Mutations in this gene are also associated with schizophrenia type 15, and are a major cause in the neurological symptoms of PMDS (9), that could be related to the severe clinical neurological features that characterize the child. While SHANK3 has been extensively studied as the cause (or at least the main contributory gene) of PMDS, many other genes are involved in the pathogenesis of this contiguous gene disorder and its phenotype. Despite the fact that SHANK3 is thought to be the strongest candidate gene for the neurological features of PMDS $(24,44,45)$, interstitial deletions of the $22 \mathrm{q} 13$ chromosomal region (that do not involve SHANK3) have rarely been detected in patients with the main clinical features common to PMDS $(46,47)$. These patients display a phenotype partially overlapping with PMDS, including developmental delay, hypotonia and language disorders, but the autism spectrum disorder symptomatology was reported less frequently in these patients (48).

Neurological impairment is a common late effect in central nervous system tumors; in contrast, it's early detection in our patient prompted genetic counseling. The diagnosis of the PMDS, a contiguous gene disorder, could explain the rapidly worsening neurological effects after cancer therapies, and the failure of multidisciplinary rehabilitation, despite the favorable trend of the oncological disease. It is possible that ring chromosome has important prognostic implications playing a role in worsening the effects of the cancer therapies, especially radiotherapy, due to the continuous risk of dynamic somatic mutations $(11,49,50)$.

Late effects due to hypothalamic-pituitary irradiation usually begins with GH deficiency. The endocrine-metabolic effects of hrGH and insulin-like growth factor-1 (IGF-1) therapy are well known in patients with pituitary defects and primary IGF-1 deficiency, respectively. In recent years, neurologic and other effects in Phelan McDermid Syndrome have been studied after IGF-1, but not after hrGH therapy. Recent evidence in mice and human neuronal models of SHANK3 deficiency $(51,52)$ suggest that IGF-1 can reverse synaptic plasticity and motor learning deficits. This evidence was also supported by Kolevzon et al. (53) in a placebo-controlled, double-blind, crossover study in which nine children with PMDS aged 5 to 15 years were treated with IGF-1or placebo for 3 months in a random order and crossed to the alternative treatment arm after a 4 week wash-out period; interestingly, during the IGF-1 therapy phase a significant improvement in both social impairment and restrictive behaviors was demonstrated. To the best of our knowledge, no studies have been conducted on recombinant human Growth Hormone (rhGH) therapy in AT/RT and PMDS and/or r22, this is the first case with GHD treated with hrGH described in the literature.

In the reported clinical case, hrGH therapy showed improvement in the growth rate despite an initial low dosage chosen for safety purposes, body thinness and spinal irradiation. It is important to underline that hrGH therapy improved the quality of life, as reported by the parents and demonstrated by a better resistance to rehabilitation stresses, recovery of dysphagia for fluids and increased muscle mass.
In consideration of the multiple procedural anesthesia, essential for radiological follow-up, the involvement of the multidisciplinary team, turned out to be crucial for reducing the risks associated with sedation. Indeed, Shank3 mutation confers enhanced sensitivity to isoflurane volatile anesthetic in central nervous systems of mutant mice models (16). This aspect deserves further study considering the important clinical implications, such as to avoid excessive use of volatile anesthetics, and suggests that this vulnerable set of patients may need additional monitoring during anesthesia.

\section{CONCLUSIONS}

At the end of this report there are some issues worth further consideration. To our knowledge, this is the first reported patient with PMDS and $\mathrm{r} 22$ that survived a childhood metastatic AT/RT. Furthermore, he is the third reported case of r22 and $22 \mathrm{q} 13$ deletion not including SMARCB1 associated to this rare malignant tumor $(8,11)$. This underlines the importance of regular screening for early detection of central nervous system rhabdoid tumors in patients with $\mathrm{r} 22$ and deletion of $22 \mathrm{q} 13$ (although not involving mutations of the SMARCB1 gene). The hrGH therapy was safe, it improved the auxological parameters and the quality of life of the entire family; however, GHD must be ruled out early when the growth velocity declines, also in the context of a normal height, in order to initiate hrGH therapy as soon as the oncological conditions allow it. The management of GHD in high-grade rhabdoid tumors is still under debate. In accordance with international guidelines, especially in the context of genetic cancer predisposition syndromes, a multidisciplinary discussion is mandatory. The GHD in cancer survivors could be treated with the same hrGH doses as is used for non-cancer GHD patients.

Finally, we would like to suggest the importance of studying and defining a protocol to reduce the potential risks related to the administration of volatile anesthetics in PMDS.

Following the analysis of Byers et al. (11), it could be argued that ring chromosome 22 may be decisive for the occurrence of AT/RT when SMARCB1 is not constitutionally mutated, however, further histogenetic analyses are necessary to explore this hypothesis, and the possible involvement of other contiguous genes.

\section{DATA AVAILABILITY STATEMENT}

The original contributions presented in the study are included in the article/supplementary material, further inquiries can be directed to the corresponding author/s.

\section{ETHICS STATEMENT}

Ethical review and approval was not required for the study on human participants in accordance with the local legislation and institutional requirements. Written informed consent to participate in this study was provided by the participants' legal guardian/next of kin. Written informed consent was obtained 
from the individual(s), and minor(s)' legal guardian/next of kin, for the publication of any potentially identifiable images or data included in this article.

\section{AUTHOR CONTRIBUTIONS}

MC and MP designed the study, collected the data, drafted and revised the manuscript. ND and $\mathrm{MG}$ took care of the patients and revised the manuscript. AV, CMi, and CMo helped in the evaluation and the following-up of the patient. VC actively participated in the data analysis, drafting, and revision

\section{REFERENCES}

1. Packer RJ, Biegel JA, Blaney S, Finlay J, Geyer JR, Heideman R, et al. Atypical teratoid/rhabdoid tumor of the central nervous system: report on workshop. J Pediatr Hematol Oncol. (2002) 24:337-42. doi: 10.1097/00043426-200206000-00004

2. Bourdeaut F, Lequin D, Brugières L, Reynaud S, Dufour C, Doz F, et al. Frequent hSNF5/INI1 germline mutations in patients with rhabdoid tumor. Clin Cancer Res. (2011) 17:31-8. doi: 10.1158/1078-0432.CCR10-1795

3. Schneppenheim R, Frühwald MC, Gesk S, Hasselblatt M, Jeibmann A, Kordes U, et al. Germline nonsense mutation and somatic inactivation of SMARCA4/BRG1 in a family with rhabdoid tumor predisposition syndrome. Am J Hum Genet. (2010) 86:279-84. doi: 10.1016/j.ajhg.2010. 01.013

4. Bookhout C, Bouldin TW, Ellison DW. Atypical teratoid/rhabdoid tumor with retained INI1 (SMARCB1) expression and loss of BRG1 (SMARCA4). Neuropathology. (2018) 38:305-8. doi: 10.1111/neup.12452

5. Rubio A. 4-year old girl with ring chromosome 22 and brain tumor. Brain Pathol. (1997) 7:1027-8. doi: 10.1111/j.1750-3639.1997.tb00902.x

6. Biegel JA. Cytogenetics and molecular genetics of childhood brain tumors. Neuro Oncol. (1999) 2:139-51. doi: 10.1215/15228517-1-2-139

7. Korones DN, Meyers SP, Rubio A, Torres C, Constine LS. A 4-year-old girl with a ventriculoperitoneal shunt metastasis of a central nervous system atypical teratoid/rhabdoid tumor. Med Pediatr Oncol. (1999) 32:389-91. doi: 10.1002/(sici)1096-911x(199905)32:5<389::aid-mpo16>3.0.co;2-h

8. Cho EH, Park JB, Kim JK. Atypical teratoid rhabdoid brain tumor in an infant with ring chromosome 22. Korean J Pediatr. (2014) 57:333-6. doi: $10.3345 / \mathrm{kjp} .2014 .57 .7 .333$

9. Sathyamoorthi S, Morales J, Bermudez J, McBride L, Luquette M, McGoey R, et al. Array analysis and molecular studies of INI1 in an infant with deletion 22q13 (Phelan-McDermid syndrome) and atypical teratoid/rhabdoid tumor. Am J Med Genet A. (2009) 149A:1067-9. doi: 10.1002/ajmg.a.32775

10. De Amorim Bernstein K, Sethi R, Trofimov A, Zeng C, Fullerton B, Yeap BY, et al. Early clinical outcomes using proton radiation for children with central nervous system atypical teratoid rhabdoid tumors. Int J Radiat Oncol Biol Phys. (2013) 86:114-20. doi: 10.1016/j.ijrobp.2012.12.004

11. Byers HM, Adam MP, LaCroix A, Leary SE, Cole B, Dobyns WB, et al. Description of a new oncogenic mechanism for atypical teratoid rhabdoid tumors in patients with ring chromosome 22. Am J Med Genet A. (2017) 173:245-9. doi: 10.1002/ajmg.a.37993

12. Cusmano-Ozog K, Manning MA, Hoyme HE. 22q13.3 deletion syndrome: a recognizable malformation syndrome associated with marked speech and language delay. Am J Med Genet C Semin Med Genet. (2007) 145C:393-8. doi: 10.1002/ajmg.c.30155

13. Kolevzon A, Angarita B, Bush L, Wang AT, Frank Y, Yang A, et al. PhelanMcDermid syndrome: a review of the literature and practice parameters for medical assessment and monitoring. J Neurodev Disord. (2014) 6:39. doi: 10.1186/1866-1955-6-39

14. Capp PL, de Faria ME, Siqueira SR, Cillo MT, Prado EG, de Siqueira JT. Special care dentistry: Midazolam conscious sedation for patients with neurological diseases. Eur J Paediatr Dent. (2010) 11:162-4. of the manuscript. All authors approved the final manuscript as submitted and agree to be accountable for all aspects of the work.

\section{ACKNOWLEDGMENTS}

The authors thank the family for their cooperation with this study; Davide Di Nitto (University of Plymouth) for helping in editing; Patrizia De Marco and Francesca Madia for genetic analyses (Array-CGH); Felice Giangaspero and Manila Antonelli for histological diagnosis and Figures 3A,B.

15. Vlassakova BG, Emmanouil DE. Perioperative considerations in children with autism spectrum disorder. Curr Opin Anaesthesiol. (2016) 29:359-66. doi: 10.1097/ACO.0000000000000325

16. Li C, Schaefer M, Gray C, Yang Y, Furmanski O, Liu S, et al. Sensitivity to isoflurane anesthesia increases in autism spectrum disorder Shank3+/ $\Delta$ c mutant mouse model. Neurotoxicol Teratol. (2017) 60:69-74. doi: 10.1016/j.ntt.2016.11.002

17. Vecht C, Royer-Perron L, Houillier C, Huberfeld G. Seizures and anticonvulsants in brain tumours: frequency, mechanisms and anti-epileptic management. Curr Pharm Des. (2017) 23:6464-87. doi: $10.2174 / 1381612823666171027130003$

18. Michaelis M, Doerr HW, Cinatl J. Valproic acid as anti-cancer drug. Curr Pharm Des. (2007) 13:3378-93. doi: 10.2174/138161207782360528

19. Judkins AR, Mauger J, Ht A, Rorke LB, Biegel JA. Immunohistochemical analysis of hSNF5/INI1 in pediatric CNS neoplasms. Am J Surg Pathol. (2004) 28:644-50. doi: 10.1097/00000478-200405000-00013

20. Judkins AR. Immunohistochemistry of INI1 expression: a new tool for old challenges in CNS and soft tissue pathology. Adv Anat Pathol. (2007) 14:3359. doi: 10.1097/PAP.0b013e3180ca8b08

21. Haberler C, Laggner U, Slave I, Czech T, Ambros IM, Ambros PF, et al. Immunohistochemical analysis of INI1 protein in malignant pediatric CNS tumors: lack of INI1 in atypical teratoid/rhabdoid tumors and in a fraction of primitive neuroectodermal tumors without rhabdoid phenotype. Am J Surg Pathol. (2006) 30:1462-8. doi: 10.1097/01.pas.0000213329.71745.ef

22. Tsai CY, Wong TT, Lee YH, Chao ME, Lin SC, Liu DJ, et al. Intact INI1 gene region with paradoxical loss of protein expression in AT/RT: implications for a possible novel mechanism associated with absence of INI1 protein immunoreactivity. Am J Surg Pathol. (2012) 36:128-33. doi: 10.1097/PAS.0b013e3182348cc4

23. Hasserjian RP, Folkerth RD, Scott RM, Schofield DE. Clinicopathologic and cytogenetic analysis of malignant rhabdoid tumor of the central nervous system. J Neurooncol. (1995) 25:193-203. doi: 10.1007/BF01053152

24. Phelan MC, Rogers RC, Saul RA, Stapleton GA, Sweet K, McDermid H, et al. 22q13 deletion syndrome. Am J Med Genet. (2001) 101:91-9. doi: 10.1002/1096-8628(20010615)101:2<91::aid-ajmg1340>3.0.co;2-c

25. Severinsen JE, Bjarkam CR, Kiaer-Larsen S, Olsen IM, Nielsen MM, Blechingberg J, et al. Evidence implicating BRD1 with brain development and susceptibility to both schizophrenia and bipolar affective disorder. $\mathrm{Mol}$ Psychiatry. (2006) 11:1126-38. doi: 10.1038/sj.mp.4001885

26. Pardiñas AF, Holmans P, Pocklington AJ, Escott-Price V, Ripke S, Carrera $\mathrm{N}$, et al. Common schizophrenia alleles are enriched in mutation-intolerant genes and in regions under strong background selection. Nat Genet. (2018) 50:381-9. doi: 10.1038/s41588-018-0059-2

27. Cai QQ, Dong YW, Qi B, Shao XT, Wang R, Chen ZY, et al. BRD1-mediated acetylation promotes integrin $\alpha \mathrm{V}$ gene expression via interaction with sulfatide. Mol Cancer Res. (2018) 16:610-22. doi: 10.1158/1541-7786.MCR-17-0527

28. Qi Q, Pan Y, Han S, Liao H, Jiang Y, Shen J, et al. PIM3 functions as oncogenic factor and promotes the tumor growth and metastasis in colorectal cancer. Anat Rec. (2019) 302:1552-60. doi: 10.1002/ar.24024

29. Marayati R, Stafman LL, Williams AP, Bownes LV, Quinn CH, Markert HR, et al. CRISPR/Cas9-mediated knockout of PIM3 suppresses tumorigenesis 
and cancer cell stemness in human hepatoblastoma cells. Cancer Gene Ther. (2021) 1-15. doi: 10.1038/s41417-021-00334-4

30. Lee EF, Harris TJ, Tran S, Evangelista M, Arulananda S, John T, et al. BCL-XL and MCL-1 are the key BCL-2 family proteins in melanoma cell survival. Cell Death Dis. (2019) 10:342. doi: 10.1038/s41419-019-1 568-3

31. Widden H, Kaczmarczyk A, Subedi A, Whitaker RH, Placzek WJ. MCL1 binds and negatively regulates the transcriptional function of tumor suppressor p73. Cell Death Dis. (2020) 11:946. doi: 10.1038/s41419-020-03068-7

32. Ridinger J, Koeneke E, Kolbinger FR, Koerholz K, Mahboobi S, Hellweg L, et al. Dual role of HDAC10 in lysosomal exocytosis and DNA repair promotes neuroblastoma chemoresistance. Sci Rep. (2018) 8:10039. doi: 10.1038/s41598-018-28265-5

33. Fan W, Huang J, Xiao H. Histone deacetylase 10 suppresses proliferation and invasion by inhibiting the phosphorylation of $\beta$-catenin and serves as an independent prognostic factor for human clear cell renal cell carcinoma. Int $J$ Clin Exp Med. (2015) 8:3734-42.

34. Li Y, Zhang X, Zhu S, Dejene EA, Peng W, Sepulveda A, et al. HDAC10 regulates cancer stem-like cell properties in KRASdriven lung adenocarcinoma. Cancer Res. (2020) 80:3265-78. doi: 10.1158/0008-5472.CAN-19-3613

35. Slaughter MJ, Shanle EK, Khan A, Chua KF, Hong T, Boxer LD, et al. HDAC inhibition results in widespread alteration of the histone acetylation landscape and BRD4 targeting to gene bodies. Cell Rep. (2021) 34:108638. doi: 10.1016/j.celrep.2020.108638

36. Ding QY, Zhang Y, Ma L, Chen YG, Wu JH, Zhang HF, et al. Inhibiting MAPK14 showed anti-prolactinoma effect. BMC Endocr Disord. (2020) 20:138. doi: 10.1186/s12902-020-00619-z

37. Cheng G, Fan X, Hao M, Wang J, Zhou X, Sun X. Higher levels of TIMP1 expression are associated with a poor prognosis in triple-negative breast cancer. Mol Cancer. (2016) 15:30. doi: 10.1186/s12943-016-0515-5

38. Sullivan I, Riera P, Andrés M, Altés A, Majem M, Blanco R, et al. Prognostic effect of VEGF gene variants in metastatic non-small-cell lung cancer patients. Angiogenesis. (2019) 22:433-40. doi: 10.1007/s10456-019-09668-y

39. Meel MH, Guillén Navarro M, de Gooijer MC, Metselaar DS, Waranecki P, Breur M, et al. MEK/MELK inhibition and blood-brain barrier deficiencies in atypical teratoid/rhabdoid tumors. Neuro Oncol. (2020) 22:58-69. doi: 10.1093/neuonc/noz151

40. Weingart MF, Roth JJ, Hutt-Cabezas M, Busse TM, Kaur H, Price A, et al. Disrupting LIN28 in atypical teratoid rhabdoid tumors reveals the importance of the mitogen activated protein kinase pathway as a therapeutic target. Oncotarget. (2015) 6:3165-77. doi: 10.18632/oncotarget.3078

41. Xiang G, Cheng Y. MiR-126-3p inhibits ovarian cancer proliferation and invasion via targeting PLXNB2. Reprod Biol. (2018) 18:218-24. doi: 10.1016/j.repbio.2018.07.005

42. Zhang Y, Shen S, Li P, Fan Y, Zhang L, Li W, et al. PLEXIN-B2 promotes the osteogenic differentiation of human bone marrow mesenchymal stem cells via activation of the RhoA signaling pathway. Cell Signal. (2019) 62:109343. doi: 10.1016/j.cellsig.2019.06.008

43. Perrot V, Vazquez-Prado J, Gutkind JS. Plexin B regulates Rho through the guanine nucleotide exchange factors leukemia-associated Rho GEF (LARG) and PDZ-RhoGEF. J Biol Chem. (2002) 277:43115-20. doi: $10.1074 /$ jbc.M206005200

44. Bonaglia MC, Giorda R, Beri S, De Agostini C, Novara F, Fichera M, et al. Molecular mechanisms generating and stabilizing terminal 22q13 deletions in 44 subjects with Phelan/McDermid syndrome. PLoS Genet. (2011) 7:e1002173. doi: 10.1371/journal.pgen.1002173

45. Luciani JJ, de Mas P, Depetris D, Mignon-Ravix C, Bottani A, Prieur M, et al. Telomeric 22q13 deletions resulting from rings, simple deletions, and translocations: cytogenetic, molecular, and clinical analyses of 32 new observations. J Med Genet. (2003) 40:690-6. doi: 10.1136/jmg.40.9.690

46. Disciglio V, Rizzo CL, Mencarelli MA, Mucciolo M, Marozza A, Di Marco $\mathrm{C}$, et al. Interstitial 22q13 deletions not involving SHANK3 gene: a new contiguous gene syndrome. Am J Med Genet A. (2014) 164A:1666-76. doi: 10.1002/ajmg.a.36513

47. Wilson HL, Crolla JA, Walker D, Artifoni L, Dallapiccola B, Takano T, et al. Interstitial 22q13 deletions: genes other than SHANK3 have major effects on cognitive and language development. Eur J Hum Genet. (2008) 16:1301-10. doi: 10.1038/ejhg.2008.107

48. Palumbo P, Accadia M, Leone MP, Palladino T, Stallone R, Carella M. et al. Clinical and molecular characterization of an emerging chromosome 22q1331 microdeletion syndrome. Am J Med Genet A. (2018) 176:391-8. doi: 10.1002/ajmg.a.38559

49. Tsilchorozidou T, Menko FH, Lalloo F, Kidd A, De Silva R, Thomas $\mathrm{H}$, et al. Constitutional rearrangements of chromosome 22 as a cause of neurofibromatosis 2. J Med Genet. (2004) 41:529-34. doi: 10.1136/jmg.2003.016774

50. Havlovicova M, Novotna D, Kocarek E, Novotna K, Bendova S, Petrak B, et al. A girl with neurofibromatosis type 1, atypical autism and mosaic ring chromosome 17. Am J Med Genet A. (2007) 143A:76-81. doi: 10.1002/ajmg.a.31569

51. Bozdagi O, Sakurai T, Papapetrou D, Wang X, Dickstein DL, Takahashi $\mathrm{N}$, et al. Haploinsufficiency of the autism-associated Shank3 gene leads to deficits in synaptic function, social interaction, and social communication. Mol Autism. (2010) 1:15. doi: 10.1186/2040-2392-1-15

52. Bozdagi O, Tavassoli T, Buxbaum JD. Insulin-like growth factor-1 rescues synaptic and motor deficits in a mouse model of autism and developmental delay. Mol Autism. (2013) 4:9. doi: 10.1186/2040-2392-4-9

53. Kolevzon A, Bush L, Wang AT, Halpern D, Frank Y, Grodberg D, et al. pilot controlled trial of insulin-like growth factor-1 in children with PhelanMcDermid syndrome. Mol Autism. (2014) 5:54. doi: 10.1186/2040-2392-5-54

Conflict of Interest: The authors declare that the research was conducted in the absence of any commercial or financial relationships that could be construed as a potential conflict of interest.

Publisher's Note: All claims expressed in this article are solely those of the authors and do not necessarily represent those of their affiliated organizations, or those of the publisher, the editors and the reviewers. Any product that may be evaluated in this article, or claim that may be made by its manufacturer, is not guaranteed or endorsed by the publisher.

Copyright $\odot 2021$ Crocco, Panciroli, Milanaccio, Morerio, Verrico, Garrè, Di Iorgi and Capra. This is an open-access article distributed under the terms of the Creative Commons Attribution License (CC BY). The use, distribution or reproduction in other forums is permitted, provided the original author(s) and the copyright owner(s) are credited and that the original publication in this journal is cited, in accordance with accepted academic practice. No use, distribution or reproduction is permitted which does not comply with these terms. 